\title{
Emittance and divergence of laser wakefield accelerated electrons
}

\author{
Christopher M. S. Sears, ${ }^{1, *}$ Alexander Buck, ${ }^{1,2}$ Karl Schmid, ${ }^{1}$ Julia Mikhailova, ${ }^{1}$ Ferenc Krausz, ${ }^{1,2}$ and Laszlo Veisz ${ }^{1, \dagger}$ \\ ${ }^{1}$ Max-Planck-Institüt für Quantenoptik, 85748 Garching, Germany \\ ${ }^{2}$ Fakultät für Physik, Ludwig-Maximilians-Universität München, 85748 Garching, Germany
}

(Received 31 May 2010; published 22 September 2010)

\begin{abstract}
We apply the pepper-pot method to measure in a single shot the transverse emittance of quasimonoenergetic electrons with $20 \mathrm{MeV}$ energy produced by laser wakefield acceleration (LWFA). The large divergence of LWFA beams ( $>1 \mathrm{mrad}$ typical) compared to conventional $\mathrm{rf}$ accelerator beams places additional restrictions on the pepper-pot design. The LWFA beam is found to have a normalized rms transverse emittance of $\epsilon_{N}=2.3 \pi \mathrm{mm} \mathrm{mrad}$, with a shot-to-shot fluctuation of $17 \%$. This emittance is comparable to state-of-the-art injectors for conventional linear accelerators. In addition, we examine the beam divergence of LWFA electrons. Simulations and theory indicate that an adiabatic reduction in the beam divergence occurs when the transition region of the downstream plasma density profile is comparable to the betatron period of the electron beam in the plasma accelerator.
\end{abstract}

PACS numbers: 06.30.Gv, 52.38.Kd

\section{INTRODUCTION}

Laser wakefield acceleration offers the promising possibility of producing high energy electrons with high peak charge in compact setups far smaller than conventional accelerators. Although first proposed many years ago [1], only relatively recently have laser wakefield accelerators succeeded in producing monoenergetic electron bunches [2-4]. Efforts since have focused on increasing the peak energy [5] and improving stability and tunability through controlled injection [6-8]. Along with these efforts, diagnostics for energy spectrum, emittance, etc. must be developed to quantify the electron beams produced. Typically techniques can be transferred from the conventional accelerator community with modifications necessary for laser wakefield acceleration. Electron energy spectrometers for laser wakefield accelerators are well developed [9-13]. Transverse emittance, however, is typically not measured in laser wakefield acceleration (LWFA) experiments.

Within the conventional accelerator community, many methods have been developed for measuring emittance. These include the quadrupole scan [14], multiscreen image analysis at different betatron phases [15,16], optical diffraction/transition radiation interference contrast (ODTRI) [17], and the pepper-pot technique [18,19]. The quadrupole scan is a multishot technique that uses variable magnetic focusing (quadrupole lenses) to scan the electron spot size at a screen located a fixed distance from the lenses in direct analogy to a beam waist scan for measuring the $M^{2}$ of a laser beam. Being multishot, the quadrupole scan requires a highly stable electron beam. It is also better suited to high energy, low charge density electron beams to limit space

\footnotetext{
*cmsears@mpq.mpg.de

†laszlo.veisz@mpq.mpg.de
}

charge effects in the electron beam focus (though space charge effects can be included in the emittance analysis [20]). For these reasons the technique is not well suited to LWFA produced electrons. The multiscreen imaging approach-involving measurement of the transverse beam profile at different positions-is also multishot since inserting any one screen will destroy the beam quality at downstream screens. It also requires a long and complicated beam transport line and a narrow energy spread electron beam to avoid chromatic effects. ODTRI, meanwhile, has a compact setup and is potentially single shot with sufficient charge, but requires the beam be at a focus in the vicinity of the radiators.

In this paper we adopt the pepper-pot technique for measuring emittance, which has the advantage of being single shot and compact. The pepper-pot method is limited in the maximum electron energy, but has been successfully applied to electron energies up to $508 \mathrm{MeV}$ [21], and is well suited in the energy range presented in this paper. Because of the large divergence of LWFA beams, extra care must be taken in specifying the slit geometry compared to beams in conventional accelerators that are typically well collimated. Even with smaller slit widths, calculation of the emittance must still take into account finite slit width to obtain an accurate measure of emittance.

The pepper-pot emittance diagnostic consists of a beam intercepting mask followed a short distance downstream by a scintillating screen (see Fig. 1). The mask is made up of an array of holes (2D) or slits (1D; used here). Strictly speaking, the term "pepper-pot" refers to the 2D hole version, though given the clear relation between the two, the word pepper-pot is commonly used to refer to either geometry. The array of holes gives emittance information in both transverse dimensions simultaneously, while the array of slits is better for low charge beams where signal at the scintillating screen is an issue. Electrons passing 


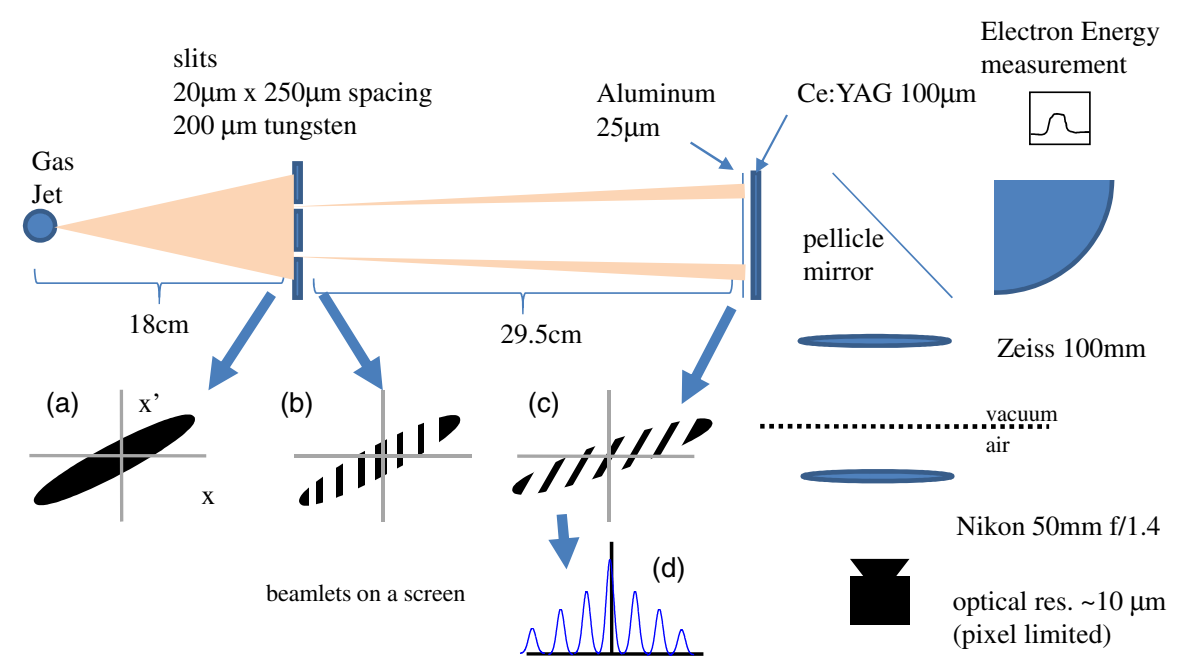

FIG. 1. Layout of the pepper-pot emittance diagnostic. Also shown is the evolution of the transverse phase space: before the mask (a), the beamlets cut out by the mask (b), after propagation in a drift (c), and the profile observed on the screen (d).

through the slits form "beamlets" that are observed on the downstream screen [Fig. 1(b)]. Those that hit the mask are scattered out of the beam. The divergence of the individual beamlets is a convolution of the collective divergence of the entire beam with the emittance spread of the electrons. The slit width must be chosen small enough to reduce the contribution from the collective divergence. This is an important issue in the case of laser wakefield produced electrons that have a large divergence $>1$ mrad. The formula for reconstructing the transverse emittance from the mask geometry and beamlet measurements on the screen is [18]

$$
\begin{aligned}
\epsilon_{x}^{2}= & \frac{1}{N^{2}}\left\{\left[\sum_{j}^{s} n_{j}\left(x_{j}-\bar{x}\right)^{2}\right]\left[\sum_{j}^{s} n_{j}\left[\sigma_{j, x^{\prime}}^{2}+\left(x_{j}^{\prime}-\bar{x}^{\prime}\right)^{2}\right]\right]\right. \\
& \left.-\left[\sum_{j}^{s} n_{j} x_{j} x_{j}^{\prime}-N \bar{x} \bar{x}^{\prime}\right]^{2}\right\} .
\end{aligned}
$$

Here $n_{j}$ is the charge of the $j^{\text {th }}$ beamlet, $x_{j}$ is the slit position, $\bar{x}$ is the mean beam position, $x_{j}^{\prime}$ is the net divergence of the $j^{\text {th }}$ beamlet, $\sigma_{j, x^{\prime}}$ is the rms divergence of the beamlet, and $\bar{x}^{\prime}$ is the mean divergence of the entire beam. The total charge is $N=\sum_{j}^{s} n_{j}$. In the case of a zero emittance beam, the beamlet size on the screen would be determined by the slit width and the collective divergence of the electrons. To estimate the angular spread due to emittance alone, we must deconvolve the slit contribution. The rms angular spread due to emittance $\sigma_{j, x^{\prime}}$ is therefore defined as

$$
\sigma_{j, x^{\prime}}^{2}=\frac{\sigma_{b m l t, j}^{2}-(M d / \sqrt{12})^{2}}{L_{s}^{2}},
$$

where $\sigma_{b l m t, j}$ is the measured rms beamlet width at the screen, $d$ is the slit width, $L_{s}$ is the slit to screen separation, and $M$ is the magnification ratio defined as $M=\left(L_{g}+L_{s}\right) / L_{g}$ with $L_{g}$ being the source to mask distance. For LWFA, the $L_{g}$ is well defined since the electrons are generated and accelerated over a short distance compared to the drift $L_{g}$. The factor of $1 / \sqrt{(}(12)$ comes from considering the rms of the flattop distribution created by the slit projection. The magnification factor $M$ accounts for the projected finite slit size increase at the screen due to the collective divergence of the electron beam. For a collimated beam, $L_{g}=\infty$ and $M=1$. Even for the small slits used in this setup, failure to account for the slit width convolution would result in a $30 \%$ overestimate of the emittance at $\epsilon_{N, r m s}=2 \pi \mathrm{mm}$ mrad. Note that through the magnification factor $M$ we have assumed the same uniformly correlated phase space for all beamlets. However, each beamlet can still shift away from a perfectly linearly correlated beam through the beamlet divergence $x_{j}^{\prime}$. Thus, this formalism can still account for nonlinear distortions to the beam phase space and the increased emittance that results.

Also note that the terms in Eq. (2) are taken in quadrature, appropriate for a convolution. A commonly applied correction appearing in the literature based on work in [19] uses a similar expression but with the terms taken linearly. As can be seen from a study of [19], this expression assumes the convolution of two flattop distributions, and gives the maximum extent of the resulting trapezoid from the convolution. This formalism is no longer valid for a Gaussian-like angular distribution nor with the width taken at half maximum rather than at the base. For two distributions with known rms widths, the width of the convolution is the square root sum of the squares of the rms widths. The use of Eq. (2) to give the deconvolved angular spread was also verified numerically by Monte Carlo simulation of the emittance measurement. 


\section{EXPERIMENTAL PEPPER-POT METHOD FOR LWFA}

Figure 1 shows the layout of the pepper-pot emittance diagnostic. The mask is made up of nine vertical slits, $2 \mathrm{~mm}$ tall, $21 \pm 2 \mu \mathrm{m}$ wide, and spaced $250 \pm 5 \mu \mathrm{m}$ apart. The slits are laser drilled in a $200 \mu \mathrm{m}$ thick tungsten plate. Tungsten is chosen to produce the maximum scatter for its thickness, having a radiation length of $3.5 \mathrm{~mm}$ [22]. $25 \mathrm{MeV}$ electrons hitting the mask are scattered by $160 \mathrm{mrad} \mathrm{rms}$ [23] producing a $5 \mathrm{~cm}$ spot at the downstream screen, much larger than the $\sim 6 \mathrm{~mm}$ projection of the slits. Thus, the scattered signal is both weak compared to the beamlets, and contributes a small nearly uniform background to the emittance image. The screen, located $29.5 \mathrm{~cm}$ from the mask, is $100 \mu \mathrm{m}$ thick Ce:YAG. Laser light is blocked by a $25 \mu \mathrm{m}$ thick aluminum sheet located less than $1 \mathrm{~mm}$ from the Ce:YAG. At this distance, additional scatter due to the aluminum produces $<8 \mu \mathrm{m}$ point spread increase which when convolved with the $>60 \mu \mathrm{m}$ beamlet size produces a negligible change. The Ce:YAG thickness is chosen to match the depth of field of the imaging system. The imaging system has a numerical aperture of 0.25 to maximize light collection and a magnification of 1.14. The resolution of the system, measured using an Edmund Optics 1951 Air Force target, is found to be pixel limited $(1 \mathrm{px}=6.45 \mu \mathrm{m})$. The imaging system also has an infinite conjugate ratio between lenses, allowing for the scintillator stage to be translated in order to optimize the signal without effecting the imaging to the camera. The entire emittance measurement assembly can also be moved out of the beam to allow undisturbed measurement of the electron beam with downstream energy and pointing diagnostics. Images are acquired by a 12 bit CCD (Point Grey Grasshopper "GRAS-14S5M") set to maximum gain.

Apart from the optical resolution of the imaging setup, we must also consider resolution limitations of the scintillator response to relativistic electrons. Direct scatter of the high energy electrons within the crystal is negligible; however, secondary electrons generated by the main beam can scatter at large angles in the crystal and are also subject to space charge forces of the primary beam. The resolution limits of Ce:YAG and other scintillators were studied by Murokh [24], who measured a $50 \mu \mathrm{m}$ spot size from a $50 \mathrm{MeV}, 250 \mathrm{pC}$ beam passing through $200 \mu \mathrm{m}$ Ce:YAG. The spot size measured by optical transition radiation for the same beam was $30 \mu \mathrm{m}$, indicating a $67 \%$ increase. Given that the crystal in our case is a factor of 2 thinner, and the total beamlet charge some 3 orders of magnitude lower, it is fair to expect the spot size increase due to effects in the Ce:YAG to be much lower. When then convolved with the $>60 \mu \mathrm{m}$ beamlet size, the emittance resolution loss should be minimal.

The electron beam is generated by laser wakefield acceleration in a helium plasma with an electron density of
$5 \times 10^{-19} \mathrm{~cm}^{-3}$ produced by a supersonic deLaval nozzle of $300 \mu \mathrm{m}$ exit diameter. The laser has a pulse energy of $80 \mathrm{~mJ}$ on-target, a pulse duration of $8 \mathrm{fs}$ [25], and is focused by a $f / 6$ off-axis parabola. Electrons are injected into the laser wake in a controlled fashion by a sharp downward density transition formed by a supersonic shockfront [26]. This shockfront is induced by inserting a razor blade into the gas flow above the nozzle. This injection mechanism improves injection probability and energy stability compared to self-injection by transverse wave breaking $[27,28]$. Generated electrons pass to the emittance diagnostic and then a small portion of the electron beam reaches the magnetic spectrometer [13]. The magnetic spectrometer has a scintillating fiber array with a sufficient sensitivity to detect and measure the energy spectrum of the subset of electrons that pass through the emittance diagnostic and reach the spectrometer.

\section{EMITTANCE MEASUREMENT RESULTS}

Figure 2 shows an example emittance measurement acquired by the diagnostic. For viewing purposes, the false color image is smoothed over the vertical direction to reduce noise. In analysis, the image is first summed vertically to produce the plot shown in the right of Fig. 2. Each beamlet is fitted with a Gaussian. The rms value of the Gaussian is used in the calculation of the emittance. Because of the low signal and high camera gain, the true rms of the beamlet signals would be dominated by pixel noise away from the peak and would prevent a reliable measure of the emittance. The inset of the right plot shows that the beamlets are well approximated by a Gaussian curve. Noise in the image increases towards the right side due to an intensity renormalization based on a careful calibration which corrects for lower light collection for off-axis points in the field of view. The renormalization scaling was obtained by acquiring an image of a uniformly illuminated field offline. Standard errors on the fit coefficients from the nonlinear curve fitting of each beamlet along with manufacture tolerances on the mask were used to calculate the total error for the emittance calculation. A detailed derivation of the error analysis can be found in [29]. The largest source of error came from beamlet charge uncertainty due to noise in the image.

The rightmost plot of Fig. 2 shows a typical electron energy spectrum acquired with the emittance diagnostic removed. Energy spectra are also acquired with the diagnostic inserted, though at much poorer resolution due to scattering. These spectra are sufficient to define the mean energy used for normalization of the emittance. About 5\% of the total charge reaches the spectrometer due to the scattering on the pepper-pot mask and other emittance diagnostic components combined with the size of the acceptance aperture of the magnetic spectrometer.

We can estimate the absolute charge from the LWFA in a two step process. First, as mentioned we observe that with 

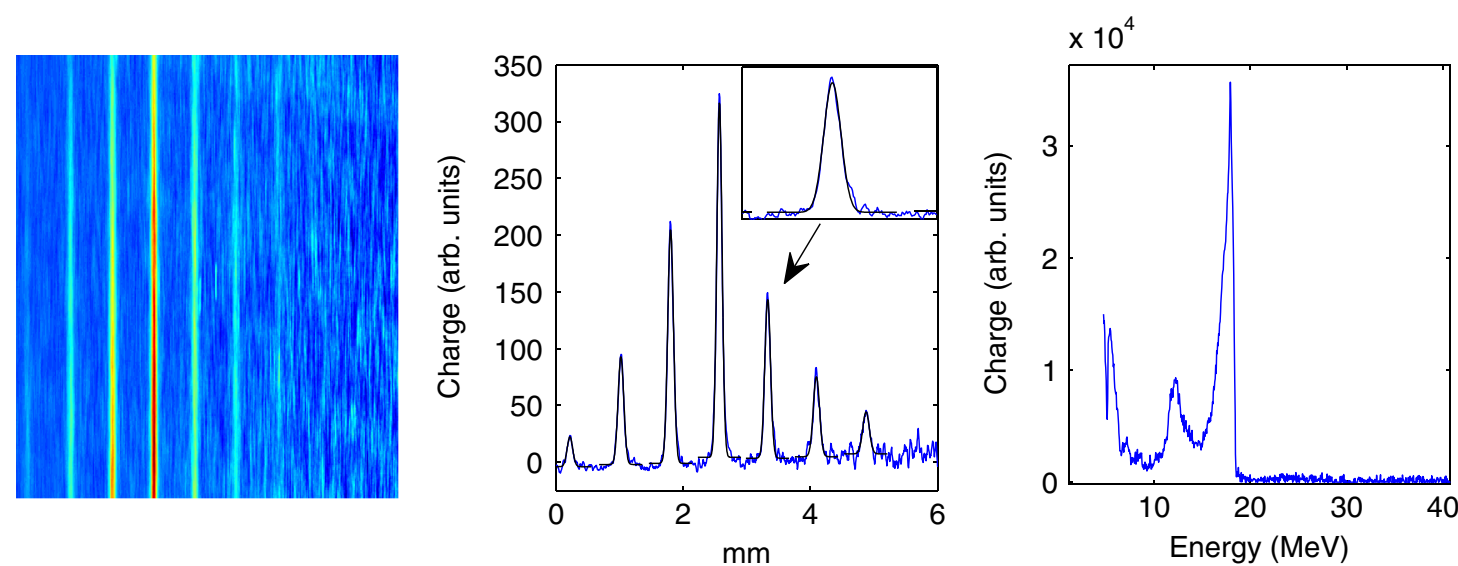

FIG. 2. Example data from the emittance diagnostic. The measured normalized rms emittance for this shot is $1.6 \pm 0.18 \pi$ mm mrad. Left: False color image at the emittance diagnostic phosphor showing the vertically stripped beamlets. Right: Vertically summed intensity across the horizontal field of view along with Gaussian fits to each beamlet. The inset shows the fit to a single peak. The mean energy for this shot was $17 \mathrm{MeV}$.

the emittance diagnostic out, the signal at the energy spectrometer is $\sim 20$ times larger than with the diagnostic inserted. With the diagnostic removed, electrons are also visible on the less sensitive but absolutely calibrated phosphor detector of the energy spectrometer [10,13]. Combining the emittance diagnostic in/out scaling factor with a cross calibration between the fiber detector and absolutely calibrated phosphor detector, we can calibrate the charge of electron spectra with the emittance diagnostic inserted. Over many shots, we obtain an average charge per

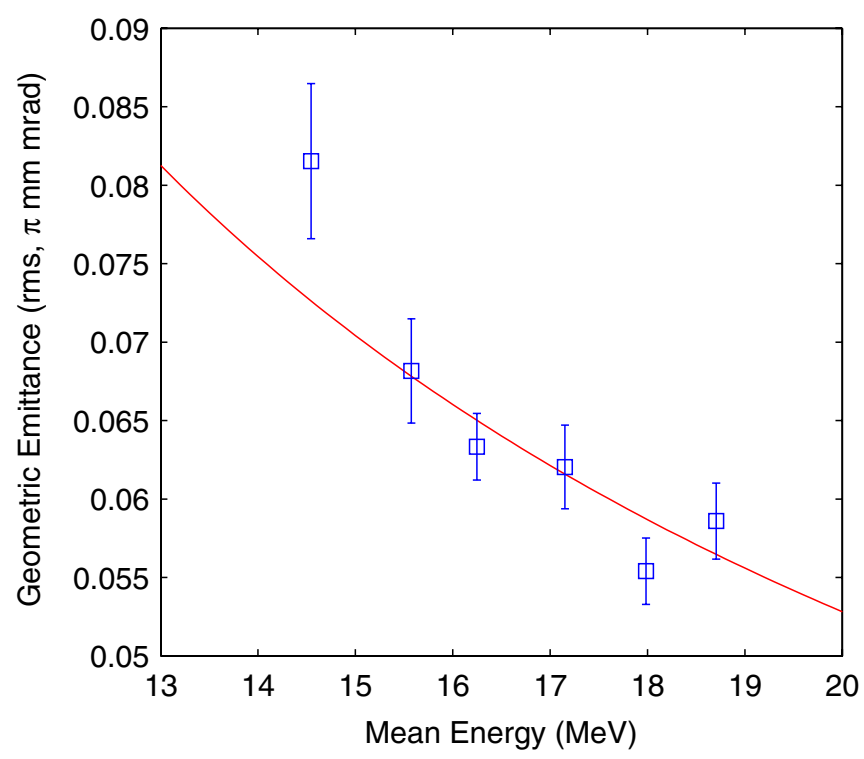

FIG. 3. Variation of the geometric emittance versus mean energy of the electrons. Error bars indicate the standard deviation of the mean for each binned set of data. The data follow a $1 / \gamma$ curve (red line) consistent with a constant normalized emittance of $2.3 \pm 17 \% \pi \mathrm{mm}$ mrad from the laser wakefield acceleration for all shots. shot of $1.2 \mathrm{pC}$ with a standard deviation of $24 \% \mathrm{rms}$. The shot in Fig. 2 has a charge of $1.0 \mathrm{pC}$.

Figure 3 shows the scaling of the geometric emittance versus mean energy of all electrons from 79 individual laser shots. The energy scaling is from the inherent jitter of the resulting electron beams-no scanning of laser or gas parameters was performed. The shots are binned according to mean energy, with the error bar indicating the standard deviation of the mean of each bin. The geometric emittance decreases with increasing mean energy, consistent with a $1 / \gamma$ scaling. This therefore indicates a constant normalized emittance from the laser wakefield acceleration. The solid line is for a normalized rms emittance of $2.3 \pi \mathrm{mm} \mathrm{mrad}$.

\section{PLASMA TO VACUUM BEAM ENVELOPE MATCHING}

The particle-in-cell (PIC) simulation [30] of the shockfront injection produces a beam with normalized emittances of 2.9 and $1.4 \pi \mathrm{mmmrad}$ [26] in the horizontal and vertical phase spaces, respectively. The horizontal emittance is larger due to heating by the laser since nearer the end of the simulation electrons catch up with the tail of the driving laser pulse. These values are in good agreement with the measured emittance above. While the electron beam is within the accelerating plasma structure, it maintains a small spot size below $1 \mu \mathrm{m}$ but with a large angular spread $>50 \mathrm{mrad}$ due to the strong focusing of the plasma wakefield [31]. This angular spread is actually substantially larger than the observed divergence of the beam downstream of the plasma accelerator. This decrease in the angular spread of the electrons is due to an adiabatic matching at the downstream plasma density roll-off of the in-channel focused spot size to the vacuum free-space divergence. Figure 4 shows how the beam angular spread 


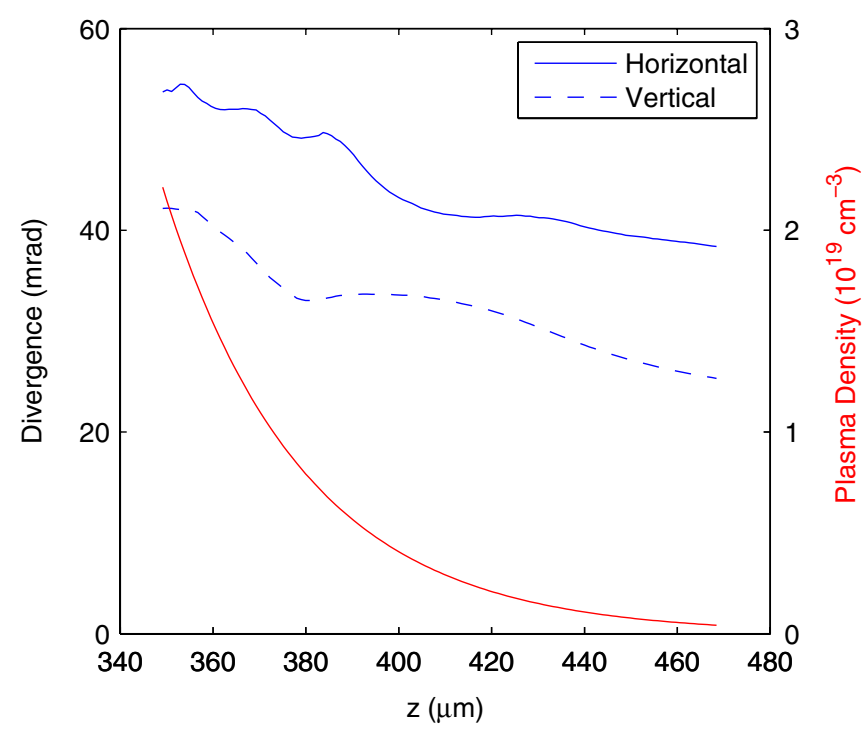

FIG. 4. Particle-in-cell simulation of the evolution of the beam angular spread at the downstream end of the plasma. Also shown is the plasma density roll-off given by an exponential function with $\sigma=30 \mu \mathrm{m}$ starting at $z=340 \mu \mathrm{m}$.

decreases during the plasma downramp. In this simulation, the downramp function is an exponential starting at a longitudinal position of $340 \mu \mathrm{m}$ with a characteristic roll-off equal to $30 \mu \mathrm{m}$. In the actual experiment, this plasma density roll-off occurs over a longer period leading to a smaller final divergence of the beam.

In addition to observing the outcoupling of the electron beam in simulation, we can gain further insight by a simple analytical model considering the transverse focusing in the plasma channel. The evolution of the beam radius in the plasma is given by [32]

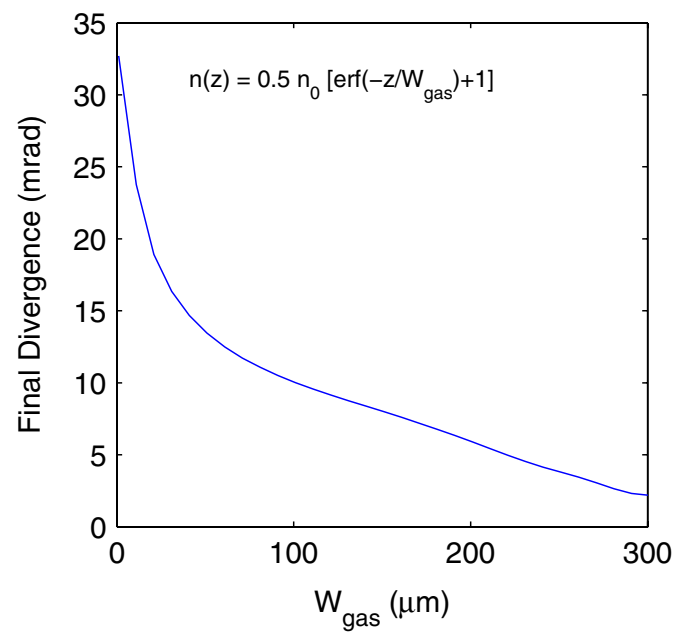

$$
\frac{d^{2} r_{b}}{d z^{2}}=\left(\frac{\epsilon_{N}}{\gamma}\right)^{2} \frac{1}{r_{b}^{3}}-\kappa_{\beta}^{2} r_{b}
$$

where $\kappa_{\beta}$ is the betatron wave number and $r_{b}$ is the beam radius. From Eq. (3) we see that the beam size is determined solely by the emittance and the focusing system (through $\kappa_{\beta}$ ). For plasma accelerators in the fully blownout regime, $\kappa_{\beta}=\kappa_{p} / \sqrt{2 \gamma}$ where $\kappa_{p}$ is the plasma wave number determined by the plasma density [31]. Therefore the roll-off in the plasma density at the downstream end can result in a weakening of the focusing strength in the channel. If the length scale of the roll-off is comparable to or greater than the betatron wavelength, the beam adiabatically expands in size while the angular spread shrinks. The total emittance is preserved and the final divergence reduced compared to a sharp plasma density transition to vacuum. The left plot in Fig. 5 shows the final divergence of a $25 \mathrm{MeV}$ electron beam from a LWFA as a function of the rate of plasma density roll-off where the plasma density profile is defined by an error function with transition width $W_{\text {plasma }}$. For a sharp truncation of the plasma density $\left(W_{\text {plasma }} \sim 0\right)$, the divergence is equal to the angular spread of the beam within the plasma accelerator. However, as the plasma profile taper increases, the final divergence of the beam decreases.

The right plot of Fig. 5 shows the particular case for the plasma profile of the nozzle used in the experiment. The final divergence of $4 \mathrm{mrad} \mathrm{rms}$ is similar to the value observed in experiment. Unfortunately, this argument can only be used as a qualitative explanation for the final divergence of an LWFA beam since the assumption of the fully blown-out plasma may not apply over the full length of the plasma density taper due to diffraction and depletion of the laser. Nevertheless, this may point to a

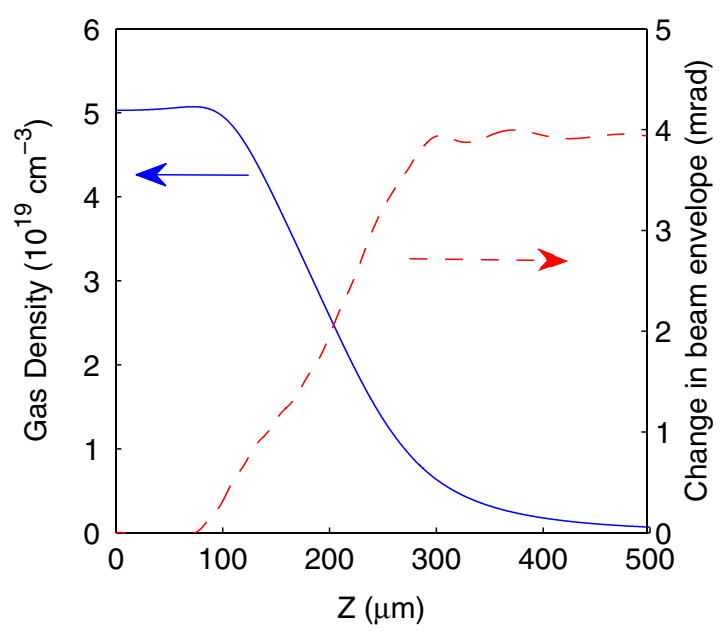

FIG. 5. Left: Outcoupled LWFA beam divergence as a function of downstream plasma density taper starting from $n_{0}=3 \times$ $10^{19} \mathrm{~cm}^{-3}$. Right: Evolution of the beam envelope derivative inside the plasma along the plasma profile of the nozzle used in the experiment. The derivative of the envelope settles to a final divergence of $4 \mathrm{mrad} \mathrm{rms}$. 
useful way of reducing the divergence of LWFA beams by controlling the exit plasma density taper.

\section{CONCLUSION}

We have measured the emittance of quasimonoenergetic electrons produced by laser wakefield acceleration utilizing injection at a steep density transition. The emittance was measured by the pepper-pot technique. The normalized rms emittance was found to be $2.3 \pi \mathrm{mm}$ mrad with a shot-to-shot jitter of $17 \%$. Careful design of the pepper-pot mask and light collection optics is vital to achieve good resolution on the emittance measurement. The large divergence of the electron beam from the wakefield also necessitates using narrow slits to reduce the contribution of the collective divergence to the beamlet size. The emittance calculation includes a correction for the finite slit width. The small emittance measured here, comparable to stateof-the-art electron sources in conventional accelerators, is an important parameter in any future light source based on LWFA produced electron beams. While the electron beam energy presented here is low, just $20 \mathrm{MeV}$, other work at conventional accelerators has demonstrated the applicability of the pepper-pot technique for energies up to $508 \mathrm{MeV}$ [21]. It is therefore reasonable to expect the pepper-pot technique to be a useful single-shot measurement for many future laser wakefield accelerators.

In this paper we have also discussed the effect of the downstream plasma taper on the outcoupling of an LWFA produced electron beam to free space. It is shown that the final divergence of the electron beam is typically less than the angular spread of electrons inside the accelerating plasma wakefield structure. This reduction comes about through an adiabatic expansion of the beam size caused by the slowly decreasing focusing strength of the plasma wake as the plasma density decreases at the downstream exit of the plasma. Note that this also increases the apparent source size. Define the source size as $\sigma_{x, s}=\epsilon_{N} / \gamma \sigma_{x^{\prime}}$, where $\sigma_{x^{\prime}}$ is the beam divergence. From the measured values here, $\epsilon_{N}=2.3 \pi \mathrm{mmmrad}, \gamma=40$, and $\sigma_{x^{\prime}}=$ $2.7 \mathrm{mrad} \mathrm{rms}$, gives an apparent source size of $21 \mu \mathrm{m}$, much larger than the laser focus and measured plasma wake structure [33]. Similarly, it is clear from this argument that combining the downstream divergence of the electron beam with the estimated beam size in the plasma channel is not a reliable way of estimating the emittance.

\section{ACKNOWLEDGMENTS}

We would like to thank Dr. Simon Jolly for useful discussions on pepper-pot emittance measurement error analysis. We would also like to thank Dr. Michael Geissler for use of his 3D PIC code, ILLUMINATION. This work is supported by DFG-Project Transregio TR18, by the Laserlab-Europe/Laptech FP7 Contract No. 228334, by the Association EURATOM-Max-Planck-Institut für
Plasmaphysik, and by The Munich Centre for Advanced Photonics (MAP). C. M. S. Sears and J. Mikhailova would also like to acknowledge the support of the Alexander von Humboldt foundation.

[1] T. Tajima and J.M. Dawson, Phys. Rev. Lett. 43, 267 (1979).

[2] S.P.D. Mangles et al., Nature (London) 431, 535 (2004).

[3] J. Faure, Y. Glinec, A. Pukhov, S. Kiselev, S. Gordienko, E. Lefebvre, J. P. Rousseau, F. Bourgy, and V. Malka, Nature (London) 431, 541 (2004).

[4] C. G. R. Geddes, C. S. Toth, J. van Tilborg, E. Esarey, C. B. Schroeder, D. Bruhwiler, C. Nieter, J. Cary, and W.P. Leemans, Nature (London) 431, 538 (2004).

[5] W. P. Leemans, B. Nagler, A. J. Gonsalves, Cs. Tóth, K. Nakamura, C. G. R. Geddes, E. Esarey, C. B. Schroeder, and S. M. Hooker, Nature Phys. 2, 696 (2006).

[6] C. G. R. Geddes, K. Nakamura, G. R. Plateau, Cs. Tóth, E. Cormier-Michel, E. Esarey, C. B. Schroeder, J. R. Cary, and W. P. Leemans, Phys. Rev. Lett. 100, 215004 (2008).

[7] T.-Y. Chien, C.-L. Chang, C.-H. Lee, J.-Y. Lin, J. Wang, and S.-Y. Chen, Phys. Rev. Lett. 94, 115003 (2005).

[8] J. Faure, C. Rechatin, A. Norlin, A. Lifschitz, Y. Glinec, and V. Malka, Nature (London) 444, 737 (2006).

[9] C. Gahn, G. D. Tsakiris, K. J. Witte, P. Thirolf, and D. Habs, Rev. Sci. Instrum. 71, 1642 (2000).

[10] A. Buck, K. Zeil, A. Popp, K. Schmid, A. Jochmann, S. D. Kraft, B. Hidding, T. Kudyakov, C. M. S. Sears, L. Veisz, S. Karsch, R. Sauerbrey, T. Cowan, F. Krausz, and U. Schramm, Rev. Sci. Instrum. 81, 033301 (2010).

[11] S. Masuda, E. Miura, K. Koyama, and S. Kato, Rev. Sci. Instrum. 79, 083301 (2008).

[12] K. Nakamura, W. Wan, N. Ybarrolaza, D. Syversrud, J. Wallig, and W. P. Leemans, Rev. Sci. Instrum. 79, 053301 (2008).

[13] C. M. S. Sears et al., Rev. Sci. Instrum. 81, 073304 (2010).

[14] M. Minty and F. Zimmermann, Measurement And Control Of Charged Particle Beams (Springer, New York, 2003).

[15] R. I. Cutler et al., in Proceedings of 12th IEEE Particle Accelerator Conference (PAC) (IEEE, Washington, DC, 1987), p. 625.

[16] Vitaly Yakimenko, Marcus Babzien, Ilan Ben-Zvi, Robert Malone, and Xijie Wang, Nucl. Instrum. Methods Phys. Res., Sect. A 483, 277 (2002).

[17] M. A. Holloway, R. B. Fiorito, A. G. Shkvarunets, P. G. O'Shea, S. V. Benson, D. Douglas, P. Evtushenko, and K. Jordan, Phys. Rev. ST Accel. Beams 11, 082801 (2008).

[18] Min Zhang, Fermilab-tm-1988, Fermi National Accelerator Laboratory, 1988.

[19] Y. Yamazaki, T. Kurihara, H. Kobayashi, I. Sato, and A. Asami, Nucl. Instrum. Methods Phys. Res., Sect. A 322, 139 (1992).

[20] S. G. Anderson, J. B. Rosenzweig, G. P. LeSage, and J. K. Crane, Phys. Rev. ST Accel. Beams 5, 014201 (2002).

[21] Nicolas Delerue et al., in Proceedings of the 23rd Particle Accelerator Conference, Vancouver, Canada, 2009 (IEEE, Piscataway, NJ, 2009). 
[22] S. Eidelman et al., Phys. Lett. B 592, 1 (2004).

[23] Max B. Reid, J. Appl. Phys. 70, 7185 (1991).

[24] A. Murokh, J. Rosenzweig, I. Ben-Zvi, X. Wang, and V. Yakimenko, in Proceedings of the 19th Particle Accelerator Conference, Chicago, Illinois, 2001 (IEEE, Piscataway, NJ, 2001), pp. 1333-1335.

[25] Daniel Herrmann, Laszlo Veisz, Raphael Tautz, Franz Tavella, Karl Schmid, Vladimir Pervak, and Ferenc Krausz, Opt. Lett. 34, 2459 (2009).

[26] Karl Schmid, Alexander Buck, Christopher M. S. Sears, Julia Mikhailova, Raphael Tautz, Daniel Harmann, Michael Geissler, Ferenc Krausz, and Laszlo Veisz, Phys. Rev. ST Accel. Beams 13, 091301 (2010).

[27] S. V. Bulanov, F. Pegoraro, A.M. Pukhov, and A. S. Sakharov, Phys. Rev. Lett. 78, 4205 (1997).
[28] Karl Schmid, Laszlo Veisz, Franz Tavella, S. Benavides, Raphael Tautz, Daniel Harmann, Alexander Buck, B. Hidding, A. Marcinkevicius, U. Schramm, M. Geissler, J. Meyer ter Vehn, D. Habs, and F. Krausz, Phys. Rev. Lett. 102, 124801 (2009).

[29] S. Jolly et al., in Proceedings of DIPAC09, Basel, Switzerland, 2009 [http://www.jacow.org/].

[30] M. Geissler et al., New J. Phys. 8, 186 (2006).

[31] E. Esarey, C. B. Schroeder, and W. P. Leemans, Rev. Mod. Phys. 81, 1229 (2009).

[32] M. Reiser, Theory and Design of Charged Particle Beams (Wiley, New York, 1994).

[33] A. Buck, M. Nicolai, K. Schmid, C. M. S. Sears, A. Sävert, J. Mikhailova, F. Krausz, M. C. Kaluza, and L. Veisz (unpublished). 